\title{
¿Medicina basada en la evidencia o Medicina centrada en el paciente $o$ ambas?
}

\author{
Evidence-based medicine or patient-centered medicine, or both?
}

Estamos en una era de la medicina en donde predominan la ciencia biológica, la información, la no tolerancia a la incertidumbre, el mercantilismo, la escasa empatía, el utilitarismo, la tecnología, etc. Estos aspectos desplazan al humanismo, una de las columnas de la medicina desde tiempos inmemoriales, y de esa forma estamos viviendo una medicina más alejada del paciente.

Karl Jaspers, médico y destacado filósofo, nos dejó la siguiente frase hace más de 50 años, que caracteriza lo que ya entonces estaba ocurriendo: "En la Medicina moderna, todo parecería estar en el mejor de los órdenes. Día a día se logran grandes resultados. Pero, lo asombroso es que en los enfermos y en los médicos aumenta la insatisfacción". ${ }^{1}$

La insatisfacción aumenta en ambos grupos sin pausa, porque los médicos perciben que su profesión ha perdido muchos de sus valores y cada vez más cuentan con menos confianza de la gente. Asimismo, los pacientes están insatisfechos porque las consultas duran pocos minutos, sin empatía y sin un juicio clínico adecuado; y para consolarlos le piden estudios auxiliares que mayormente son innecesarios.

El manejo de los síntomas es una piedra angular de la atención clínica, especialmente para pacientes con afecciones crónicas. Sin embargo, los médicos no detectan en la atención del paciente los síntomas,sus discapacidades físicas y sus preocupaciones. En EE.UU., esto sucede hasta en la mitad de las consultas, especialmente las realizadas en las clínicas. ${ }^{2}$

Probablemente, con el fin de que la medicina mejorase, hace más de 25 años surgió la Medicina basada en la evidencia (MBE), que sitúo como paradigma el ensayo clínico aleatorizado (ECA). ${ }^{3}$ Esta metodología surgió por primera vez en 1948 cuando los doctores D'Arcy Hart y Bradford Hill de Inglaterra, asignaron aleatoriamente a enfermos de tuberculosis dos tratamientos, estreptomicina y placebo. ${ }^{4}$ Este estudio permitió que la estreptomicina salvara la vida de muchísimos pacientes al cambiar sustancialmente la supervivencia en enfermos tuberculosos.

Con los ECA, comenzaba una nueva era junto con la evidencia y estudios de observación de alta calidad, con los conocimientos y la experiencia de los profesionales. Sin duda, la MBE ha generado múltiples beneficios a los pacientes a través de investigaciones bien conducidas, de muy alta calidad y apropiadas en diferentes poblaciones, acorde lo que se observa en las revisiones sistemáticas bien realizadas.

No obstante, la MBE tiene varias debilidades al confiar abiertamente en los ECA siendo que la mayoría de las investigaciones no se prueban en entornos clínicos y poblaciones heterogéneas. Como ejemplo, vemos que en la práctica los médicos corren el riesgo de extrapolar estimaciones de una población para hacer el diagnóstico de pacientes individuales. "Hay muchos pacientes parecidos, pero todos los pacientes son diferentes y aunque dos pacientes presenten una misma enfermedad, muestren los mismos sintomas, y sus pruebas de laboratorio sean idénticas, se tratará de dos pacientes distintos" (Sacristán J.). ${ }^{3}$ Algo similar sería el antiguo refrán "no hay enfermedades, hay pacientes".

Es notorio que las poblaciones estudiadas son distintas que los pacientes individuales, y por tanto la MBE despierta la duda acerca de que si los datos de los resultados en los ECA podrían orientar las decisiones en pacientes individuales. A pesar de los éxitos de la MBE, la importante variabilidad en su implementación sigue siendo un problema de magnitud.

Asimismo, hay otros factores externos que están creciendo y desvirtúan francamente los principios fundacionales de la MBE, que ha motivado en años recientes un cierto desprestigio. Greenhalgh et al. señalan que hay una crisis porque principalmente ha sido invadida por intereses comerciales, en especial, las industrias farmacéutica y de dispositivos médicos. El gran volumen de evidencia, ya es inmanejable, y su calidad ha sido apropiada y distorsionada por intereses particulares. La industria señala la dosis de los fármacos de intervención y de control, y publica los resultados en las principales revistas. Según Greenhalgh, la MBE pasó en los últimos años de investigar y tratar enfermedades a detectar e intervenir en no-enfermedades. La verdadera evidencia debe ser personalizada para el paciente y no limitada por normas e intereses. ${ }^{5}$

Es de señalar también que es imprescindible que la evidencia vaya de la mano con la incertidumbre, que es lo único que nos permitirá reflexionar si nuestro juicio clínico es correcto o no. La incertidumbre es inevitable cuando 
aplicamos la evidencia procedente de ECA de poblaciones, pero no de individuos.

Iona Heath, en un ensayo manifiesta la necesidad de que los médicos conozcan la evidencia con indicaciones claras de sus limitaciones y que a través de la incertidumbre, detecten el posible daño; y agrega que "esto animaría a los clínicos a pensar en vez de decirles qué hacer". ${ }^{6}$

La MBE nos hace describir a las personas en términos de datos de ciencia biomédica, pero estos no son, y nunca serán, suficientes. Tal evidencia es esencial, pero siempre insuficiente para el cuidado de los pacientes.

Abbasi, en un editorial titulado "Necesitamos más humanidad, y mejor evidencia", señala que la obsesión con la evidencia disminuye nuestra humanidad en las consultas clínicas y cita a Iona Heath, que dice "para ofrecer consultas coherentes y equilibradas, necesitamos un nuevo enfoque, a fin que los médicos puedan cerrar la brecha entre evidencia y humanidad".?

Las limitaciones de la evidencia se recogen claramente en el estudio de Nagendranet et al., que examinaron si los resultados de los ECA con efectos grandes se replicaban en estudios de seguimiento. Salvo que el tamaño del efecto en el ECA tuviera un riesgo relativo $\geq 20$, el estudio de seguimiento no mostró efectos significativos. ${ }^{8}$

Respecto a la Medicina centrada en el paciente (MCP), surgió hace más de 20 años luego de la MBE. Su deseo fue reivindicar la necesidad de volver la mirada hacia el paciente individual, entendido como persona. ${ }^{3} \mathrm{~A}$ lo largo de la historia muchos médicos ilustres preconizaron esta visión y es muy probable que William Osler, un excepcional clínico y gran humanista, fuera el precursor de lo que 90 años después tomarían los que diseñaron la MBP. Una sabia frase de las tantas que Osler nos dejó y que apoya la MBP dice "si no fuera por la gran variabilidad entre los individuos, la medicina bien podría ser una ciencia y no un arte". Solo con estas palabras está destacando que el arte es esencial en la medicina y la variabilidad nuestra hace que las evidencias sean muchas veces insuficientes para aplicarlas sin reflexionar

Millenson, señala que la MBP nació como un marco conceptual que "adopta conscientemente la perspectiva del paciente", y enfatiza que actualmente, los cambios tecnológicos, económicos y sociales están llevando la atención médica en direcciones impensables por aquellos pioneros del enfoque en el paciente. Para Milenson, hoy la MBP está siendo parte de un conjunto más amplio bajo fuerzas más grandes de intereses económicos, en una medicina cada vez más mercantilizada donde el lucro se extiende constantemente. $^{9}$

Como vemos, ambas formas de medicina se están deteriorando por los perjudiciales cambios que invaden a nuestra profesión con poderosos intereses basados solo en el lucro.

No obstante, actualmente hay un interés que la MBE y la MBP se unan para que los médicos y pacientes puedan evaluar la evidencia de las investigaciones y exigir una medicina más personalizada. Esa unión podría ser beneficiosa para lograr investigaciones adecuadas a la necesidad de los pacientes y que se orienten hacia un abordaje poblacional. ${ }^{3}$

Es probable que solo así se logren mejores resultados en el cuidado de la salud y que las investigaciones de evidencias eviten problemas que puedan perjudicar a los pacientes, que obviamente no lo merecen ya que sin duda, los médicos debemos siempre velar por ellos.

\section{José María Ceriani Cernadas}

Editor

http: / / dx.doi.org/10.5546/ aap.2018.90

Texto completo en inglés:

http: / / dx.doi.org/10.5546/ aap.2018.eng.90

Cómo citar: Ceriani Cernadas JM. ¿Medicina basada en la evidencia o Medicina centrada en el paciente o ambas? Arch Argent Pediatr 2018;116(2):90-91.

\section{REFERENCIAS}

1. Jaspers K. La práctica médica en la era tecnológica. Barcelona: Gedisa;1988.

2. Basch E. Patient-Reported Outcomes- Harnessing Patients' Voices to Improve Clinical Care. $N$ Engl J Med. 2017;376(2):106-8.

3. Sacristán JA. Medicina basada en la evidencia y medicina centrada en el paciente: algunas reflexiones sobre su integración. Rev Clin Esp. 2013;213(9):460-4.

4. Medical Research Council Streptomycin in Tuberculosis Trials Committee. Streptomycin treatment for pulmonary tuberculosis. Brit Med J. 1948;2(4582):769-83.

5. Greenhalgh T, Howick J, Maskrey N. Evidence based medicine: a movement in crisis? BMJ. 2014;348:g3725.

6. Heath I. How medicine has exploited rationality at the expense of humanity: an essay by Iona Heath. BMJ. 2016;355:i5705.

7. Abbasi K. We need more humanity as well as better evidence. BMJ. 2016;355:i5907.

8. Nagendran M, Pereira TV, Kiew G, et al. Very large treatment effects in randomised trials as an empirical marker to indicate whether subsequent trials are necessary: metaepidemiological assessment. BMJ. 2016;355:i5432.

9. Millenson ML. When "patient centred" is nolonger enough: the challenge of collaborative health. BMJ. 2017;358:j3048. 